\title{
Formation of terrestrial planets from planetesimals around M dwarfs
}

\author{
Masahiro Ogihara and Shigeru Ida \\ Department of Earth and Planetary Sciences, Tokyo Institute of Technology, \\ 2-12-1-I2-10 Ookayama, Meguro-ku, Tokyo 152-8551, Japan \\ email: ogihara@geo.titech.ac.jp, ida@geo.titech.ac.jp
}

\begin{abstract}
We have investigated accretion of terrestrial planets from planetesimals around M dwarfs through N-body simulations including the effect of tidal interaction with disk gas. Because of low luminosity of $\mathrm{M}$ dwarfs, habitable zones around them are located near the disk inner edge. Planetary embryos undergo type-I migration and pile up near the disk inner edge. We found that after repeated close scatterings and occasional collisions, three or four planets eventually remain in stable orbits in their mean motion resonances. Furthermore, large amount of waterrich planetesimals rapidly migrate to the terrestrial planet regions from outside of the snow line, so that formed planets in these regions have much more water contents than those around solar-type stars.
\end{abstract}

Keywords. accretion, accretion disks, methods: n-body simulations, planetary systems: formation

\section{Introduction}

In the past about 10 years, over 250 extrasolar planets have been discovered mainly with radial velocity surveys. Although $\mathrm{M}$ dwarfs account for about $70-80 \%$ of stars in the galactic disk, their low luminosity is disadvantageous for spectroscopic observation, so that radial velocity surveys have not discovered large number of planets around $\mathrm{M}$ dwarfs. However, as improvement of spectroscopic observations and development of gravitational microlensing survey (its detection efficiency is independent of stellar luminosity), planetary systems around M stars are being revealed. Around M dwarfs, Jupiter-mass gas giants are generally rare (e.g., Johnson et al. 2007), but Neptune-mass planets are rather abundant (e.g., Beaulieu et al. 2006), compared with solar-type stars. Lower disk mass and lower disk temperature may account for these properties (Ida \& Lin 2005). Due to the low luminosity of $\mathrm{M}$ dwarfs, habitable zones are close to the host stars. It allows for detection of habitable planets by radial velocity observation. In fact, the first detected potentially habitable planet is orbiting an M dwarf (Udry et al. 2007). On the other hand, the first detected transiting Neptune-sized planet is GJ 436b that orbits an M dwarf (e.g., Gillon et al. 2007). The evaluated internal density suggests that the planet is composed mainly of ice, in spite of its proximity to the host star.

Ida \& Lin (2005) theoretically studied planetary systems around M dwarfs, extending their model based on solar-type stars. Although their prediction on formation and retention rates of gas giants and Neptune-mass planets is consistent with observational data, their model may not provide detailed information on formation of planets in habitable zones. Since habitable zones are much closer to host stars for M dwarfs than for solartype stars, the formation process of habitable planets may not be directly extrapolated from that around solar-type stars. Raymond et al. (2007) performed N-body simulations of terrestrial planet formation from planetary embryos around low-mass stars and found that terrestrial planets in the habitable zones of most $M$ dwarfs are likely to be small 
and dry. However, they neglected the effects of protoplanetary disk gas. In such inner regions, gas density is so high that both gas drag and tidal interaction are very effective, and accretion timescale of terrestrial planets would be much shorter than disk lifetime. Thereby, we carry out N-body simulations including the tidal damping of orbital eccentricity, inclination and semimajor axis due to disk gas.

When we include the effect of type-I migration, which is caused by imbalance between tidal interaction with inner disk gas and that with outer one (e.g., Goldreich \& Tremaine 1980, Ward 1986), the proximity of habitable zones to inner disk edge would play an important role in the formation of habitable planets around M dwarfs, because type-I migration stops at the edge and planets would accumulate there. Terquem \& Papaloizou (2007) performed N-body simulations of planetary embryos undergoing type-I migration. They found that the migrating embryos originally formed at $\sim 1$ AU are trapped one after another in mean motion resonances by the preceding embryos near the disk inner edge to form two to five resonant close-in planets. In the case of $\mathrm{M}$ dwarfs, the original formation regions of embryos are very close to the accumulation regions, so that the trapped planets would be perturbed by the embryos that emerge nearby.

\section{Model}

We consider a host star with $0.2 M_{\odot}$ and set the locations of disk inner edge and snow line at $0.05 \mathrm{AU}$ and $0.3 \mathrm{AU}$, respectively. We adopt the minimum mass solar nebula model (Hayashi 1981) for the surface density of the disks. Initially, 5,000 planetesimals with mass $3.0 \times 10^{23} \mathrm{~g}$ (inside of the snow line) and $1.7 \times 10^{24} \mathrm{~g}$ (outside of snow line) are placed between $0.05 \mathrm{AU}$ and $0.4 \mathrm{AU}$.

The equations of motions of particle $k$ at $\boldsymbol{r}_{k}$ in heliocentric coordinates are

$$
\frac{d^{2} \boldsymbol{r}_{k}}{d t^{2}}=-G M_{*} \frac{\boldsymbol{r}_{k}}{\left|\boldsymbol{r}_{k}\right|^{3}}-\sum_{j \neq i} G M_{j} \frac{\boldsymbol{r}_{k}-\boldsymbol{r}_{j}}{\left|\boldsymbol{r}_{k}-\boldsymbol{r}_{j}\right|^{3}}+\boldsymbol{F}_{\mathrm{grav}}+\boldsymbol{F}_{\mathrm{gas}}
$$

where $k, j=1,2, \ldots$, the first term is gravitational force of the central star and the second term is mutual gravity between the bodies. The self-gravity, which is the most expensive part in calculation, is calculated with GRAPE-6 $\boldsymbol{F}_{\text {grav }}$ is specific gravitational drag force due to tidal interaction with disk gas and $\boldsymbol{F}_{\text {gas }}$ is specific aerodynamical gas drag force. These formulas are derived by Tanaka \& Ward (2004) through linear analysis. The physical radius of a body is determined by its mass and internal density. We adopt realistic value $3 \mathrm{~g} \mathrm{~cm}^{-3}$ for internal density. When physical sizes of bodies overlap, perfect accretion is assumed. After the collision, a new body is created, conserving total mass and momentum of the two colliding body.

\section{Results}

A typical result is shown in Figure 1. In the early stage of planetary accretion, larger planetesimals grow faster than smaller ones, resulting in runaway growth of the planetesimals. As the runaway bodies (embryos) grow, orbital decay due to tidal interaction with disk gas (type I migration) starts. At the disk inner edge, the migrations cease and they interact with preceding planets. Since embryos emerge one after another, the planets repeat close scatterings with them and occasionally merge with them. However, they end up in stable orbits captured in their mean motion resonances. In this case, three planets in mean motion resonances remain. The final configuration of the three planets is shown in Figure 2. The pair of the innermost planet and the second one has a $3: 2$ 


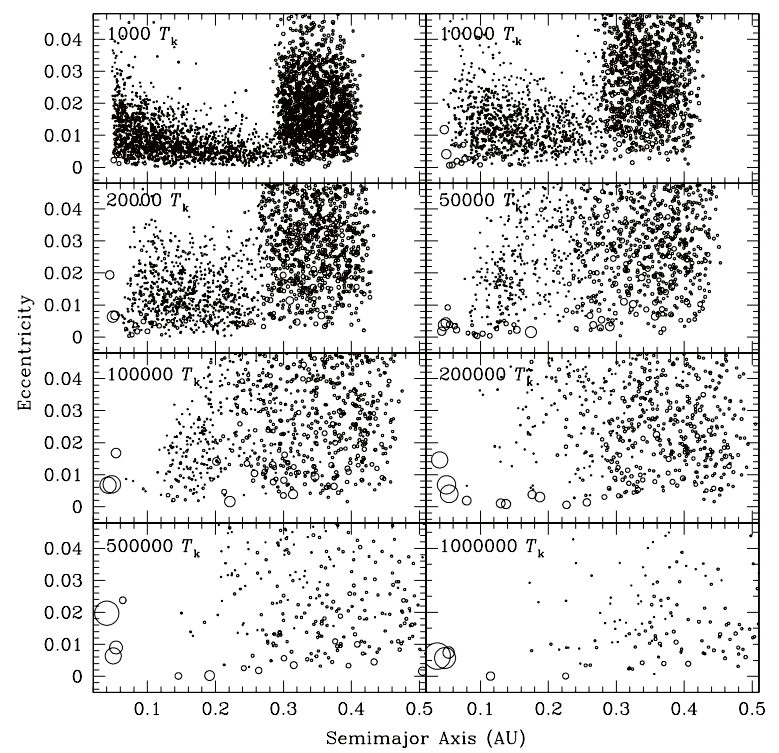

Figure 1. Time evolution of planetesimals. $a$ and $e$ are semimajor axis and eccentricity. Size of circles are proportional to physical radii of the bodies. Snow line and disk inner edge are located at $0.3 \mathrm{AU}$ and $0.05 \mathrm{AU}$, respectively. $T_{\mathrm{k}}$ is the kepler time at $0.1 \mathrm{AU}$ around a $0.2 M_{\odot}$ star.

orbital period commensurability (resonance) and that of the second and third ones is in a $7: 6$ resonance. The most massive planet is the innermost one, the mass of which is about $4 M_{\oplus}$.

In other runs, we find similar final results: three or four planets captured in mutual resonances in the disk inner edge. Even for different disk models (gas and planetesimals), this result does not change, although final planets are generally larger for more massive planetesimal disks. We take out the final planets and integrate their orbits until $10^{6}$ years with gas surface density decaying exponentially and found that their orbital configuration is stable after the decay of disk gas.

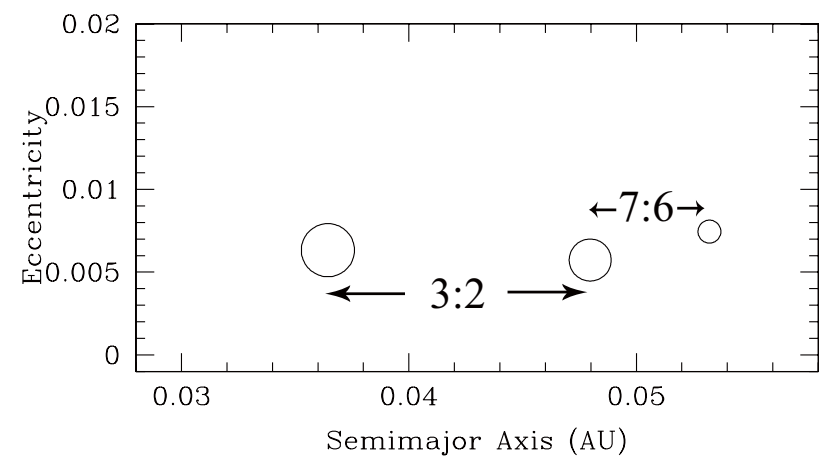

Figure 2. Final configuration of the system near the disk inner edge. The circles represent the planets. Commensurate relationships between orbital period are also shown. 


\section{Discussion}

We have investigated accretion of terrestrial planets from planetesimals around $\mathrm{M}$ dwarfs through N-body simulations. We incorporated the effect of gas disk into N-body simulation. We found that three or four resonant planets are formed near the disk edge after successive close scatterings and merging. The result is similar to the case of solar type stars examined by Terquem \& Papaloizou (2007). The improved radial velocity observation from ground or transit observation from space such as Corot or Kepler will test this prediction.

In our simulation, we also found that the close-in resonant planets are composed mostly of planetesimals that have migrated from the regions beyond the snow line. This result is in contrast to that obtained by Raymond et al. (2007) that neglected type-I migration. In comparison with solar-type stars, habitable planets around $\mathrm{M}$ dwarfs would contain much water. This is because growth timescale of planetary embryos and their migration timescale are much shorter than disk lifetime even outside the snow line for the case of $\mathrm{M}$ dwarfs. The low probability of formation of gas giants around $\mathrm{M}$ dwarfs allows for retention of icy planetesimals and it also facilitates supply of icy planetesimals to inner regions.

\section{References}

Beaulieu, J.-P., Bennett, D. P., Fouqué, P., Williams, A., Dominik, M., Jørgensen, U. G., Kubas, D., Cassan, A., Coutures, C., Greenhill, J., Hill, K., Menzies, J., Sackett, P. D., Albrow, M., Brillant, S., Caldwell, J. A. R., Calitz, J. J., Cook, K. H., Corrales, E., Desort, M., Dieters, S., Dominis, D., Donatowicz, J., Hoffman, M., Kana, S., Marquette, J. -B., Martin, R., Meintjes, P., Pollard, K., Sahu, K., Vinter, C., Wambsganss, J., Woller, K., Horne, K., Steele, I., Bramich, D. M., Burgdorf, M., Snodgrass, C., Bode, M., Udalski, A., Szymański, M. K., Kubiak, M., Wieckowski, T., Pietrzyński, G., Soszyński, I., Szewczyk, O., Wyrzykowski, Ł., Paczyński, B., Abe, F., Bond, I. A., Britton, T. R., Gilmore, A. C., Hearnshaw, J. B., Itow, Y., Kamiya, K., Kilmartin, P. M., Korpela, A. V., Masuda, K., Matsubara, Y., Motomura, M., Muraki, Y., Nakamura, S., Okada, C., Ohnishi, K., Rattenbury, N. J., Sako, T., Sato, S., Sasaki, M., Sekiguchi, T., Sullivan, D. J., Tristram, P. J., Yock, P. C. M., \& Yoshioka, T. 2006, Nature, 439, 437

Gillon, M., Pont, F., Demory, B.O., Mallmann, F., Mayor, M., Mazeh, T., Queloz, D., Shporer, A., Udry, S., \& Vuissoz, C. 2007, A\& $A, 472$, L13

Goldreich, P. \& Tremaine, S. 1980, ApJ, 241, 425

Hayashi, C. 1981, Prog. Theor. Phys. Suppl., 70, 35

Ida, S. \& Lin, D.N.C. 2005, ApJ, 626, 1045

Johnson, J. A., Butler, R. P., Marcy, G. W., Fischer, D. A., Vogt, S. S., Wright, J. T., \& Peek, K. M. G. 2007, ApJ, 670, 833

Raymond, S. N., Scalo, J., \& Meadows, V. S. 2007, ApJ, 669, 606

Tanaka, H. \& Ward, W. R. 2004, ApJ, 602, 388

Terquem, C. \& Papaloizou, J. C. B. 2007, ApJ, 654, 1110

Udry, S., Bonfils, X., Delfosse, X., Forveille, T., Mayor, M., Perrier, C., Bouchy, F., Lovis, C., Pepe, F., Queloz, D., \& Bertaux, J. L. 2007, A\&\&A, 469, L43

Ward, W. R. 1986, Icarus, 67, 164 